\title{
Uncertainty among Scottish psychiatrists over NHS continuing care guidance
}

\author{
AIMS AND METHOD \\ To examine knowledge among \\ Scottish psychiatrists of the 1996 \\ guidance on National Health Service \\ (NHS) responsibility for continuing \\ healthcare, a postal questionnaire \\ was devised and sent to the 132 con- \\ sultants and specialist registrars in \\ the South-East Scotland Deanery in \\ May 2007.
}

\author{
RESULTS \\ Two-thirds of the clinicians (67\%, \\ $n=88$ ) responded, $88 \%$ of whom had \\ in-patient responsibilities. Only $24 \%$ \\ responded positively to an awareness \\ of the current Scottish guidance. \\ There was a strong association \\ between awareness of the guidance \\ and psychiatric speciality, and \\ greater awareness among clinicians \\ with previous experience of a \\ relevant complaint or appeal.
}

\begin{abstract}
CLINICAL IMPLICATIONS
Uncertainty about NHS continuing healthcare guidance among practising psychiatrists suggests a need for increased awareness of the issues, with training implications for the new guidance on NHS continuing healthcare in Scotland which became effective in February 2008.
\end{abstract}

Section 1 of the National Health Service (Scotland) Act 1978 outlines the general duty of the Secretary of State (now the Scottish Ministers) to promote a comprehensive and integrated health service and to provide or secure the effective provision of services for that purpose, whereas Section 36 relates to the provision of accommodation and services, including medical and nursing, to meet all reasonable requirements under that Act. Each National Health Service (NHS) Board in Scotland has a duty to ensure any necessary arrangements are organised for in-patients prior to discharge, the decision to discharge being a clinical one made by the doctor responsible for the patient's care. Additionally, each Board has a duty to meet the healthcare needs of people in its geographical area who require NHS continuing healthcare, commonly referred to as NHS continuing care, which can be provided in a variety of settings but is paid for by the NHS Boards. In 1996 the Scottish Office Department of Health issued a circular (NHS MEL (1996) 22, referred to here as MEL 22), entitled NHS Responsibility for Continuing Health Care, that set out the responsibilities of the NHS both to take decisions about discharge of patients from hospital care and to secure NHS continuing care where required. ${ }^{1}$ This document stated that NHS continuing care may be provided in a hospital, a hospice or in a nursing home or other appropriate facility. It also contained a procedure enabling patients to appeal against discharge from NHS hospital care.

Similar guidance was issued elsewhere in the UK, but the situation in England and Wales has since developed owing to judgments by the Court of Appeal and the High Court in England, and reports issued by the Health Services Ombudsman for England in 2003. ${ }^{2}$ These developments attracted media attention, as a result of which the NHS in Scotland received a number of complaints about the funding of continuing care, leading the Scottish Executive Health Department's Directorate of Service Policy and Planning to issue guidance on the handling of such complaints and reaffirming MEL 22 as the appropriate guidance on NHS continuing care in Scotland. ${ }^{3}$ The Scottish Public Services Ombudsman (SPSO) who raised concerns regarding the legitimacy of the application of MEL 22 investigated a number of complaints about decisions regarding discharge from NHS continuing care in Scotland. ${ }^{4}$ The SPSO also raised the issue about whether decisions by English courts might be expected to have a bearing on policy and practice in Scotland, raising this issue with the Scottish Executive Health Department, and recommending an urgent need to review the MEL 22 guidance issued more than a decade earlier. ${ }^{4}$ In October 2007, the Department of Health for England and Wales implemented updated guidelines concerning NHS continuing healthcare eligibility criteria that are linked to a 'primary need for healthcare' and provided a decision-support tool to assist in the process. ${ }^{5,6}$ The eligibility assessment is needs-based and is carried out by the multidisciplinary team in line with the core values and principles set out in the National Framework. ${ }^{5}$ It was in this climate that this study aimed to investigate the knowledge base of Scottish psychiatrists with clinical responsibilities for in-patient care about the application of the MEL 22 guidance.

\section{Method}

A questionnaire based on the MEL 22 guidance relating to discharge and to appeals against discharge from NHS continuing care in Scotland was devised and piloted among 15 psychiatrists in the West Scotland Deanery. The modified anonymised questionnaires, consisting of 19 structured multiple choice questions (a total of 31 questions) were posted in May 2007 to the 132 psychiatric consultants and specialist registrars in the South-East Scotland Deanery. Three questions related to clinical status and experience of managing in-patient care within the past decade, three related to professional experience of the appeal or complaints procedure, and the remaining questions were based on awareness and knowledge of the MEL 22 guidance and regulations. 
A postal reminder was sent to non-responders after 6 weeks, after which the data were collated and

original papers analysed. We used SPSS version 13.0 for Windows and Microsoft Excel software for the data analyses, with twosample t-tests. Results were considered to be significant at the $5 \%$ level.

\section{Results}

Two-thirds (88 clinicians) of the original sample of 132 responded to the survey, of whom $82 \%$ were at consultant grade and $17 \%$ at specialist registrar grade. Overall, $46 \%$ were in general adult psychiatry, $26 \%$ in psychiatry of older adults, learning disability and rehabilitation (POA/LD/Rehab) specialties and $26 \%$ in other psychiatric specialties. The survey response rate from POA/LD/Rehab specialties was $80 \%$ compared with $62 \%$ from general psychiatry and $64 \%$ from other specialties. The response rate from consultants was $68 \%$ compared with $60 \%$ for specialist registrars.

Eighty-eight per cent of respondents held clinical responsibilities for in-patient care currently or during the past decade. Although overall $24 \%$ reported an awareness of current guidance on NHS responsibility for continuing care, $65 \%$ in the POA/LD/Rehab specialties were aware of the guidance compared with only $8 \%$ in general psychiatry and $9 \%$ in other subspecialties. Overall, $14 \%$ reported familiarity with the MEL 22 guidance document ( $44 \%$ of POA/LD/Rehab psychiatrists, none of the general psychiatrists and $4 \%$ of the others). Seven respondents stated being aware of the current guidance but were unfamiliar with MEL 22. Eighty-two per cent were uncertain if guidance on NHS continuing care differed between Scotland and England.

The knowledge of the respondents was investigated further by asking a series of 23 questions. Of the 88 respondents, 2 did not answer these questions. The highest number of correct responses was 19 out of 23 (2 respondents) with 10 respondents achieving just one correct answer. Table 1 summarises the distribution of these knowledge scores. Clinicians with responsibility for in-patient care in the past decade had significantly higher scores than those without $(P=0.003)$. On average, POA/ LD/Rehab psychiatrists answered significantly more $(52 \% ; P<0.005)$ of the 23 questions correctly, compared with 34\% for general psychiatrists and 31\% for other subspecialties. None of the 18 respondents with a knowledge score less than 20\% reported having awareness of current guidelines. Of the 15 with a score of $60 \%$ or more, 7 were familiar with MEL 22 but equally 7 claimed to have no familiarity with it (and 1 was uncertain). Consultants were able to answer $40 \%$ of the awareness questions correctly compared with $32 \%$ of specialist registrars, a non-significant difference $(P=0.174)$.

Forty-four per cent of respondents accurately identified that the decision to discharge from NHS continuing care, after relevant assessments had been completed, was primarily one for the consultant responsible for the patient. The responses as to why a patient might require NHS continuing care are shown in Table 2 ,
Table 1. Distribution of MEL 22 knowledge scores

\begin{tabular}{ccc}
$\begin{array}{c}\text { Questions } \\
\text { answered } \\
\text { correctly, \% }\end{array}$ & $\begin{array}{c}\text { Respondents } \\
n\end{array}$ & $\begin{array}{c}\text { respondents } \\
\%\end{array}$ \\
\hline $0-20$ & 18 & 20.5 \\
$21-40$ & 29 & 33.0 \\
$41-60$ & 26 & 29.5 \\
$61-80$ & 13 & 14.8 \\
$81-100$ & 2 & 2.3 \\
\hline
\end{tabular}

with Table 3 indicating appropriate care settings for NHS continuing care as stated in MEL 22. The majority of respondents (68\%) were aware that NHS continuing care need not be permanent, with $61 \%$ aware that patients could appeal against the decision to discharge from NHS care.

The majority of appeals and complaints relating to NHS continuing care issues involved clinicians in the POA/ LD/Rehab specialist groups. All 9 of the respondents with experience of an appeal against discharge from NHS continuing care and all 7 of those with experience of investigating a complaint about discharge from NHS continuing care were from this group. Five of the nine respondents who had dealings with the SPSO's office

Table 2. Percentage of respondents identifying each statement as one of the criteria for NHS continuing healthcare $(n=88)$

Reasons for NHS continuing care Yes, \%

Requirement for ongoing and regular clinical

supervision

Complexity, nature or intensity of the patient's

health needs

Frequent and not easily predictable clinical

interventions

Routine use of specialist healthcare equipment

Treatments that require the supervision of

specialist NHS staff

Rapidly degenerating or unstable condition

requiring specialist medical or nursing supervision Ineligibility for benefits on discharge from hospital ${ }^{\text {a }}$ Terminally ill and requires specialist palliative

healthcare

Death is imminent

NHS, National Health Service.

a. The only reason in this table not qualifying as a criterion for NHS continuing care.

\section{Table 3. Correct responses to locality for NHS continuing care} $(n=88)$

Place of NHS continuing care Yes, \%

Hospital 53

Care home 49

Supported housing

Patient's own home

Other setting

NHS, National Health Service. 
were also in this group (the others consisted of two from general psychiatry and two from other specialties).

Although $10 \%$ of respondents had been professionally involved in such an appeal, the majority (82\%) were uncertain of time limits to appeal submission, and only 11 $(13 \%)$ correctly identified that the Director of Public Health initially investigates the appeal, with similar numbers (14\%) correctly recording that an independent clinician from a different health board has a key role in a second-stage appeal process. There was uncertainty (77\%) as to whether the second-stage appeal outcome was binding on all parties, with only $26 \%$ identifying that there are further avenues of redress in unsuccessful appeals against discharge from NHS continuing care. Ten per cent of the responding clinicians indicated that they had been involved clinically and/or professionally with the SPSO. There were significantly higher 'knowledge scores' among clinicians who had been involved in these discharge appeals $(P=0.005)$ or complaints $(P=0.032)$, but not for those involved clinically or professionally with the SPSO when compared with the remaining clinicians.

\section{Discussion}

This is a relatively large survey of senior psychiatrists in south-east Scotland, with a response rate of $66 \%$, of whom $88 \%$ had clinical responsibility for NHS in-patient care during the period that the MEL 22 guidance applied. The findings suggest that many consultants and specialist trainees were not fully aware of the MEL 22 guidance or of the appeal processes within it. Although the results may be skewed by the greater response rate from the $\mathrm{POA} / \mathrm{LD} /$ Rehab group, and to a lesser extent by the slightly higher response rate from the consultants, any response bias from this would probably overestimate the levels of knowledge among psychiatrists overall. The majority of respondents were able to correctly answer $20-60 \%$ of the questions relating to the guidance, but relatively few demonstrated a thorough knowledge.

There is a strong association between subspecialty and awareness of the regulations. As expected perhaps, awareness was greater among groups of doctors who were most likely to have patients who more often meet the needs for NHS continuing care, particularly psychiatry of older adults and rehabilitation, and among those who had previous experience of appeals or complaints in relation to NHS continuing care. A similar picture appears to exist in relation to familiarity with the actual MEL 22 guidance, corroborated by the knowledge scores. If these findings are typical of other parts of Scotland, this suggests that there is a section of the psychiatric profession with a good awareness of the correct procedures although unaware of the specific guidance.

In response to concerns raised by the SPSO and other bodies, the Scottish Government Health Department in February 2008 issued CEL 6 (2008), NHS Continuing Healthcare, which provided guidance replacing that of MEL 22. ${ }^{7}$ The criteria for NHS continuing healthcare in Scotland are essentially unchanged from MEL 22, but other changes include a stronger emphasis on record-keeping, clearer communication with patients and carers, and the appeal process. The newer guidance indicates that, as with the Department of Health guidance, a decision support tool will be developed and piloted to support decision-making, but that ultimately the decision about NHS continuing healthcare in Scotland will remain a judgement of the consultant (or general practitioner in some community hospitals).

In Scotland, NHS Boards are now in the process of implementing the CEL 6 (2008) guidance, with an initial 12 -month period when the application of the guidance will be monitored by the Scottish Government Health Department. Given the findings of our survey, it is recommended that clinicians with responsibility for in-patient care in the NHS be fully informed of the new guidance, of the local processes for implementing the guidance as they are developed, and how the guidance may affect their service responsibilities. Relevant information should be accessible and included in future induction and training programmes for higher trainees and clinicians. Finally, unlike the MEL 22 guidance which lacked national reporting or monitoring of routine implementation, a national system should be devised and operationally responsible for overseeing and auditing the CEL 6 (2008) guidance to ensure consistent and best practice across the Scottish NHS Boards.

\section{Acknowledgements}

Andrew Elders, senior information analyst with NHS Fife, provided statistical advice and carried out analyses of the data.

\section{Declaration of interest}

None.

\section{References}

1 The Scottish Office. NHS Responsibility for Continuing Health Care. NHS MEL (1996): 22. Department of Health 1996.

2 Health Service Ombudsman. NHS Funding for Long Term Care. TSO (The Stationery Office), 2003 (http://www.ombudsman.org. uk/pdfs/care03.pdf).

3 Scottish Executive. Handling Complaints About the Funding of Continuing Health Care. Health Department Directorate of Service Policy and Planning, 2003.

4 Scottish Public Services Ombudsman. Ombudsman's CommentaryJune 2007. SPSO 2007 (http://www.spso.org.uk/ investigation-reports/2007/ june/ombudsmans-commentaryjune-07).

5 Department of Health. The National Framework for NHS Continuing Healthcare and NHS-funded Nursing Care. Department of Health, 2007 (http://www.dh.gov. uk/en/Publicationsandstatistics/ Publications/PublicationsPolicyAnd Guidance/DH_076288).

6 Department of Health. DecisionSupport Tool for NHS Continuing Healthcare. Department of Health 2007 (http://www.dh.gov.uk/en/ SocialCare/Deliveringadultsocial care/Continuingcare/DH_073912).

7 Scottish Government. NHS Continuing Healthcare. CEL 6 Care Directorate, 2008. (2008). Primary and Community

*Gary S. Stevenson Consultant Psychiatrist, NHS Fife, Department of Psychiatry, Stratheden Hospital, Cupar, Fife KY15 5RR, email: garystevenson@ fife-pct.scot.nhs.uk, Agnieszka Philipson Specialist Training Year 4 in Old Age Psychiatry, South East Scotland Psychiatric Rotation, Gordon McLaren Consultant in Public Health Medicine, NHS Fife, Cameron Hospital, Fife original papers 